\title{
Innovación docente para el aprendizaje de la configuración electrónica de un elemento en alumnos de Educación Primaria
}

\section{Teaching innovation for learning the electronic configuration of an element in Primary Education students}

BelÉN Begines Ruiz

ORCID: https://orcid.org/0000-0002-1513-7443

Departamento de Química

Orgánica y Farmacéutica

Universidad de Sevilla

bbegines@us.es

DOI: http://dx.doi.org/10.12795/9788447231003.004

Pp.: 86-106 


\section{Introducción}

Este ciclo de mejora en el aula (CIMA) se aplica en la asignatura de Fundamento de Ciencias de la Materia, impartido en primero del Grado en Educación Primaria y del Doble Grado de Educación Infantil y Primaria, así como en cuarto del Doble Grado en Educación Primaria y Estudios Franceses y en tercero del Doble Grado en Lengua y Literatura Alemanas y en Educación Primaria. El contexto de este curso está marcado por la situación sanitaria derivada de la covid-19, lo que ha significado que los alumnos han recibido la docencia online, dificultando la interacción entre ellos mismos y con el docente. Además, ha mermado la asistencia a clase, ya que podían encontrar las sesiones grabadas en la plataforma para oírlas a su conveniencia. Esto se ha traducido en una reducción del número de asistentes a las sesiones, que se ha reducido a unos 40 estudiantes, que son los que han trabajado durante el CIMA.

\section{Diseño previo del CIMA}

\section{Mapa de contenidos y problemas claves}

El CIMA se diseñó para tratar parte de los contenidos del tema 2 de la asignatura, enfocado en la estructura del átomo. Estos contenidos se muestran en la Figura 1, los cuales se abordan desde 4 problemas diferentes presentados a los estudiantes. Estos problemas intentan abarcar específicamente contenidos del tipo Conceptos, Datos, Procedimientos Intelectuales y Procedimientos Psicomotrices (Porlán, R., 2017), todos ellos necesarios para entender la estructura del átomo. Pero, además, de forma transversal a estos contenidos, se pretende trabajar también un contenido de tipo Actitudinal, basado en el fomento del rigor científico, intentado mostrar a los estudiantes la importancia de razonar, de preguntarnos el por qué de las cosas $y$, sobre todo, de trabajar de manera ordenada, siendo metódico en nuestra forma de proceder.

Ciclos de Mejora en el Aula (2020). Experiencias de Innovación Docente de la US Esta obra se distribuye con la licencia Creative Commons 


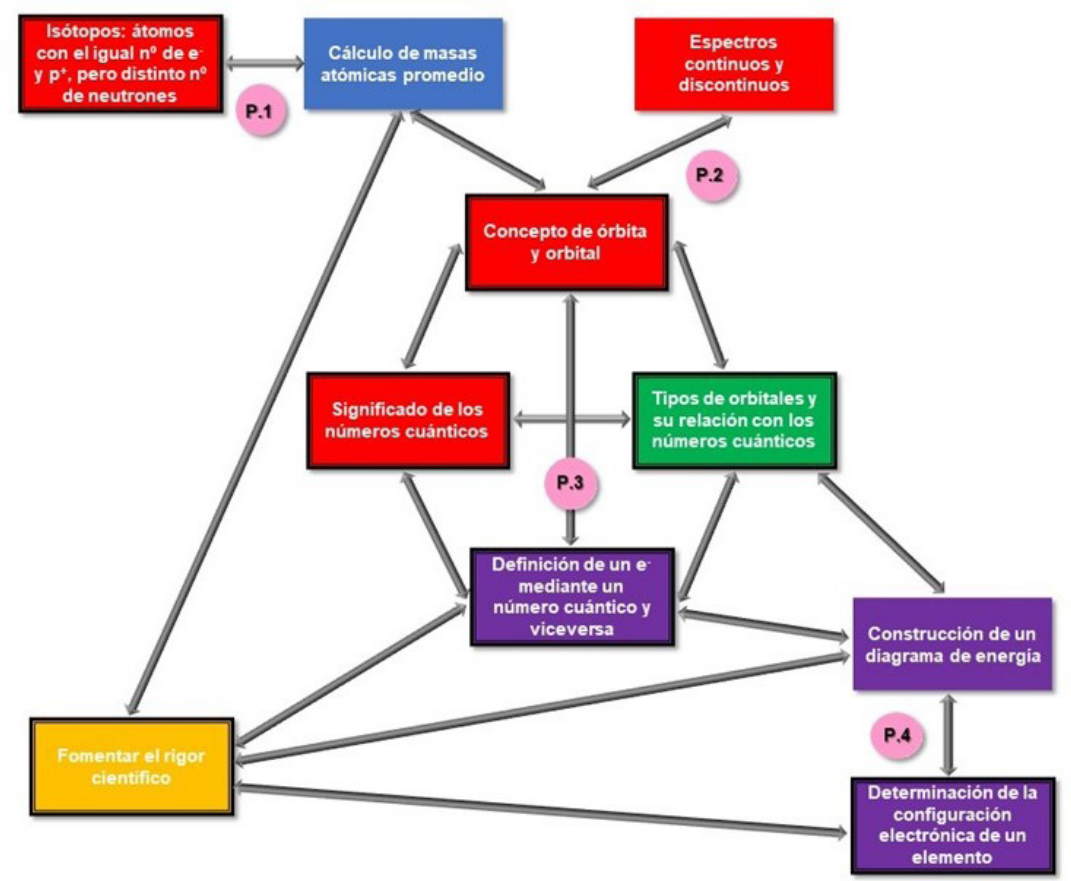

TIPOSDE CONTENIDOS
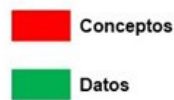

Procedimientos Intelectuales

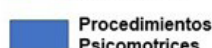
Psicomotrices

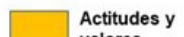
valores

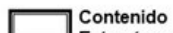
Estructurante

Problema planteado a los alumnos

\section{PROBLEMAS}

P.1 ¿Cómo se explica que la masa del cloro sea 35,45 uma?

P.2 ¿Qué diferencias hay entre espectro continuo y discontinuo?, ¿Por qué se generan los espacios discontinuos?

P.3 ¿Cómo debemos aplicar los números cuánticos para diferenciar los distintos electrones que tiene un átomo y poder, así, diferenciarlos?

P.4 ¿Cómo podemos representar de una vez todos los electrones de un átomo?

Figura 1. Mapa de contenidos a tratar en este CIMA, incluyendo los problemas planteados a los estudiantes

\section{Modelo metodológico posible}

Mi modelo metodológico intenta seguir el esquema presentado en la Figura 2 y está basado en las ideas de Bain (2008), Finkel (2000) y Porlán (2017). Se apoya fundamentalmente en el trabajo inicial del alumno, que debe

Ciclos de Mejora en el Aula (2020). Experiencias de Innovación Docente de la US (c) (i) $\Theta$ Esta obra se distribuye con la licencia Creative Commons 
intentar dar respuesta a un problema/pregunta, seguido de una puesta en común de las hipótesis a las que hayan llegado con su trabajo inicial. Por último, pretende que los alumnos pongan en tela de juicio su modelo mental a través de las preguntas/ejercicios de contraste.

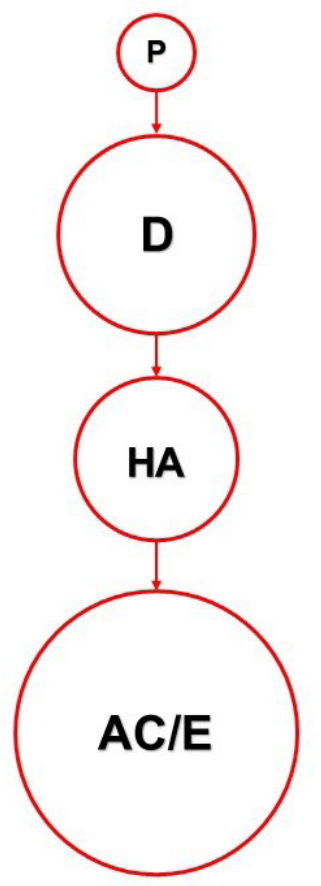

P: Planteamiento de un problema

D: Discusión

HA: Hipótesis de los alumnos

AC/E: Actividades de contraste o

ejercicios planteados como actividades

de contraste

Figura 2: Modelo metodológico posible

\section{Cuestionario inicial-final}

El cuestionario inicial incluye 4 preguntas correspondientes a los 4 problemas que se plantearán a los alumnos, aunque se expresan con un lenguaje más coloquial enfocado a que el estudiante entienda bien la pregunta. Además, lleva incluido un contexto creado para atraer a los alumnos:

Nos encontramos a principios del siglo XX. Como editora principal de la revista Nature, estoy preparando una edición especial de la revista encaminada a resolver algunas

Ciclos de Mejora en el Aula (2020). Experiencias de Innovación Docente de la US Esta obra se distribuye con la licencia Creative Commons 
de las incógnitas que actualmente se están generando alrededor del concepto de átomo y de la distribución de los electrones alrededor de su núcleo. Por ello, estoy buscando grupos de investigación de distintas universidades de prestigio que quieran participar en este proyecto. Si estáis interesados en formar parte de estos grupos de investigación, necesito conocer si estáis realmente preparados y motivados para la tarea que se os va a encomendar. Por favor contestad a esta pequeña encuesta con vuestras palabras. Tenéis que convencerme de que podéis participar, por lo tanto, argumentar bien vuestras opiniones

1. ¿Cómo calcularías la masa atómica en un átomo de un elemento si no tuvieras ese dato en la tabla periódica?

2. ¿Cómo se mueven los electrones alrededor del núcleo?

3. ¿Qué nomenclatura usaríais para definir un determinado electrón de un átomo para poder diferenciarlo de otro electrón del mismo átomo?

4. ¿Crees que todos los electrones de un átomo tienen la misma energía? Razona tu respuesta.

Por supuesto, el cuestionario final lleva las mismas 4 preguntas.

\section{Secuencia de actividades}

La idea general en la que se basa el diseño del CIMA es plantear el problema en la clase anterior para que los alumnos puedan trabajarlo/discutirlo en casa y que en la clase correspondiente expongan sus hipótesis, a la vez que yo voy haciendo preguntas de contraste. En determinados problemas, he usado ejercicios prácticos como actividades de contraste, para seguir trabajando las mismas preguntas ya lanzadas durante el debate. Además, he intentado plantear problemas bastante concretos que se puedan tratar en una o dos sesiones.

Ciclos de Mejora en el Aula (2020). Experiencias de Innovación Docente de la US Esta obra se distribuye con la licencia Creative Commons 
Tabla 1. Secuencia de actividades del Problema 1: ¿Cómo se explica que la masa del cloro sea 35,45 uma?

\begin{tabular}{|c|c|c|c|}
\hline \multicolumn{4}{|c|}{ SESIÓN 1} \\
\hline 1 & Presentación del problema & P.1 & $10^{\prime}$ \\
\hline \multicolumn{4}{|c|}{$\begin{array}{l}\text { Antes de empezar la experiencia habré creado yo grupos de 5-6 alumnos, } \\
\text { intentando que contengan todos el mismo número de alumnos que hayan } \\
\text { estudiado Ciencias en el bachillerato. Cada grupo pertenecerá a una } \\
\text { afamada universidad y estará compuesto por un catedrático, un profesor, } \\
\text { un doctorando, un estudiante de máster, un estudiante de grado y un } \\
\text { técnico de laboratorio. Los roles los asignaré yo, en función también de } \\
\text { sus estudios previos, para que todos los alumnos que tengan el mismo rol } \\
\text { posean, a su vez, una base de conocimiento similar. Puesto que formamos } \\
\text { un comité de expertos de principios del siglo XX, el editor de la prestigiosa } \\
\text { revista científica Nature nos pide que expliquemos algunos de los enigmas } \\
\text { que aún existen en el campo de la Química. Por supuesto, sólo publicará, } \\
\text { y por tanto adquirirá fama mundial, aquella universidad que explique más } \\
\text { claramente los enigmas que nos plantea la revista. El primer enigma que } \\
\text { nos pide que expliquemos es el siguiente: ¿Cómo se explica que la masa } \\
\text { del cloro sea 35,45 uma? }\end{array}$} \\
\hline \multicolumn{4}{|c|}{ Recursos: Foto de la revista Nature } \\
\hline 2 & Debate en grupo & D & $15^{\prime}$ \\
\hline \multicolumn{4}{|c|}{$\begin{array}{l}\text { Los alumnos debatirán en grupo para conseguir el nuevo modelo atómico. } \\
\text { Les comunicaré que deben ir anotando las conclusiones a las que vayan } \\
\text { llegando puesto que después tendrán que exponerlas. En este caso será el } \\
\text { catedrático de cada grupo el que presente las conclusiones de su grupo. } \\
\text { Yo intervendré sólo en el caso en el que me lo soliciten. Mientras estaré } \\
\text { pasando de una sala a otra dentro de la EV para comprobar que todo vaya } \\
\text { bien }\end{array}$} \\
\hline \multicolumn{4}{|c|}{ Recursos: Ninguno en clase, generación de una sala por grupo en EV } \\
\hline 3 & Exposición de resultados & HA & $15^{\prime}$ \\
\hline
\end{tabular}

Ciclos de Mejora en el Aula (2020). Experiencias de Innovación Docente de la US Esta obra se distribuye con la licencia Creative Commons Reconocimiento-NoComercial-SinObraDerivada Internacional (CC BY-NC-ND 4.0.) 
Se debatirá en clase las conclusiones a las que han llegado los distintos grupos, anotándolas en la pizarra. Los encargados de exponerlas serán los catedráticos. Inicialmente, preguntaré sus conclusiones a un solo grupo. Una vez éste haya terminado, iré preguntando a cada catedrático si están de acuerdo con esas conclusiones y si su grupo ha llegado a alguna otra. Si no van saliendo como conclusiones, yo les haré las siguientes preguntas: ¿Qué diferencia a un tipo de elemento de otro? ¿Todos los elementos tienen el mismo número de protones? ¿Y de neutrones? ¿Cómo calcularíamos la masa de un átomo de un elemento del que conocemos su A? Pero si existen átomos de un mismo elemento que poseen distinto no de neutrones y, por tanto, distinta masa atómica, ¿cómo calcularíamos entonces su masa atómica promedio?

Recursos: Ninguno

\begin{tabular}{|l|l|l|l|}
\hline 4 & Ejercicios de contraste & AC/E & $30^{\prime}$ \\
\hline
\end{tabular}

Realizaremos los ejercicios 4, 5 y 6 del boletín de problemas. Estos serán realizados por los profesores presentes en clase, que explicarán cómo los resuelven. En estos ejercicios se presentan casos de elementos que presentan distintos isótopos y se les pide a los alumnos que calculen su masa atómica promedio, realizando el ejercicio inverso al que se le presentó como problema: en vez de explicar el motivo de una masa promedio final con decimales, debida a la presencia de isótopos, ahora, partiendo de los isótopos, deben calcular esa masa atómica promedio

Recursos: Boletín de ejercicios

Tabla 2. Secuencia de actividades del Problema 2: ¿Qué diferencias hay entre espectro continuo y discontinuo?, ¿por qué se generan los espacios discontinuos?

\begin{tabular}{|l|l|l|l|}
\hline 5 & Presentación del problema & P.2 & $10^{\prime}$ \\
\hline Cuando comiencen esta actividad, los alumnos ya conocen que un átomo \\
de un elemento está compuesto por protones, neutrones y electrones. \\
Hace muy poco que Ernest Rutherford propuso su modelo atómico, un \\
modelo que dice que el núcleo atómico concentra todos los protones y \\
neutrones del átomo mientras que los electrones se encuentran girando \\
alrededor del núcleo. Pero acabamos de leer un artículo científico donde \\
se habla de la existencia de los espectros discontinuos. Para darle aún más \\
prestigio a nuestra universidad, deberemos ser los primeros en proponer \\
un nuevo modelo atómico que sea capaz de justificar la generación de \\
estos espectros discontinuos. \\
Como actividad entregable, cada grupo debe enviar su propuesta de \\
modelo atómico y los motivos que los lleva a proponerla, respondiendo \\
a las siguientes preguntas: ¿Qué diferencias entre espectro continuo y \\
espectro discontinuo? ¿Por qué se generan los espectros discontinuos? \\
\hline
\end{tabular}

Ciclos de Mejora en el Aula (2020). Experiencias de Innovación Docente de la US Esta obra se distribuye con la licencia Creative Commons 
Recursos: Ninguno

ENTRE LA SESIÓN 1 Y LA 2

\begin{tabular}{|l|l|l}
\hline 1 & Debate en grupo & D \\
\hline
\end{tabular}

Los alumnos debatirán en grupo para conseguir responder a las preguntas planteadas en el problema 2

Recursos: En esta situación sanitaria, los que los alumnos planteen para poder organizar las reuniones virtuales

\section{SESIÓN 2}

\begin{tabular}{|l|l|l|l|}
\hline 1 & $\begin{array}{l}\text { Recuerdo del problema presentado } \\
\text { anteriormente }\end{array}$ & P.2 & $10^{\prime}$ \\
\hline
\end{tabular}

Se les recordará a los alumnos el problema presentado en la sesión anterior y sobre el que han debido trabajar entre sesiones, respondiendo las siguientes preguntas: ¿Qué diferencias entre espectro continuo y espectro discontinuo? ¿Por qué se generan los espectros discontinuos?

Recursos: Ninguno

\begin{tabular}{|l|l|l|l|}
2 & Exposición de resultados & HA & $15^{\prime}$ \\
\hline
\end{tabular}

Se debatirá en clase las conclusiones a las que han llegado los distintos grupos, anotándolas en la pizarra. Los encargados serán los doctorandos.

Recursos: Ninguno

3 Preguntas de contraste

$A C$
$30^{\prime}$

Como preguntas de contraste, iré introduciendo, si ellos no lo han dilucidado aún: ¿Cómo se mueve el electrón alrededor del átomo? ¿Se mueve siempre en la misma órbita? ¿Podemos calcular la distancia de esta órbita al núcleo? Si el electrón se encuentra en estado excitado, ¿Qué ocurre cuando vuelve a su estado fundamental? Entonces, ¿cuál es el motivo de la generación de los espectros atómicos? ¿Qué diferencia hay entre un espectro discontinuo y uno continuo?

Estas preguntas de contrate se irán haciendo, si procede, durante la exposición de los resultados o ya al final, si fuera necesario

Recursos: Una transparencia con la representación de los saltos de los e- y su relación con los espectros de emisión, sólo al final del debate

Los problemas 3 y 4 se diseñan de forma similar a los anteriormente expuestos, variando únicamente el tiempo invertido en las actividades de contraste. De hecho, para los problemas 3 y 4 se usan, además de preguntas de contraste, una serie de ejercicios.

Ciclos de Mejora en el Aula (2020). Experiencias de Innovación Docente de la US Esta obra se distribuye con la licencia Creative Commons 


\section{Aplicación del CIMA}

\section{Diario de las sesiones}

- Sesión del 09/11/2020: De nuevo, los problemas técnicos me llevaron a comenzar la sesión con más de 35 minutos de retraso, lo que hizo que ya fuera con mucho estrés porque no me iba a dar tiempo a llevar a cabo todo lo que tenía planeado. Sin embargo, la sesión fue bastante bien, ayudado por el hecho de que el problema planteado era relativamente sencillo, así, con los 15 minutos de debate en grupo que tuvieron fueron suficientes para que la gran mayoría de la clase captara los conceptos que se pretendía que adquirieran. Seleccioné a la Harvard University para que su técnico de laboratorio expusiera sus conclusiones. Este alumno fue capaz de explicar bastante bien el concepto de isótopos y lo que ello conlleva respecto al número de protones y neutrones. Aun así, yo hice algunas preguntas de contraste como: ¿Todos los elementos tienen el mismo número de protones? ¿Y de neutrones? ¿Qué característica diferencia a los distintos elementos? Una vez tuvieron bien claro esto, yo les mostré cómo se representa un elemento, lo que me sirvió para nombrarles el número másico. Como actividad de contraste, les puse varios ejemplos de elementos y les fui preguntando para que me indicaran el número atómico y el número másico, así como toda la información extra que pudieran obtener. Al final, pareció que todos habían entendido bastante bien los conceptos asociados a los isótopos. La nota negativa fue que el hecho de empezar la clase tan tarde me restó el tiempo necesario para que hiciéramos la actividad 4 planteada para ese día. Sólo pudimos debatir (entre toda la clase) cómo calcularíamos la masa atómica promedio de un elemento teniendo en cuenta sus isótopos. Además, tampoco pude plantearles

Ciclos de Mejora en el Aula (2020). Experiencias de Innovación Docente de la US 
el problema siguiente, aunque les dije que se lo plantearía por correo, cosa que hice a la mañana siguiente.

En general, aun habiendo tenido los problemas técnicos, estoy muy satisfecha por cómo evolucionó la sesión. En el momento en que la terminé tenía ciertas dudas de la opinión que tenían los alumnos respecto al cambio de metodología. Pero estas dudas se disiparon completamente cuando recibí al día siguiente 15 correos de 15 alumnos pidiéndome participar en la actividad. Esto era un 50\% más de alumnos. Lo cual me hace pensar que les gustó bastante. Ojalá sea así...

- Sesión del 12/11/2020: Antes de comenzar la sesión, yo había leído todas las actividades presentadas por todas las universidades. Sorpresivamente, casi todas habían sido capaces de explicar bastante bien la diferencia entre espectro continuo y discontinuo, que era la parte que más me preocupaba a mí. Y casi todos habían explicado bastante bien la generación de los espectros continuos, de forma que muchas de las preguntas que yo tenía como preguntas de contraste ya habían sido resueltas por ellos. Aun así, cuando comenzó la clase y una vez recordado el problema, seleccioné a los catedráticos, como representantes del día de cada universidad, y a la universidad que había presentado una actividad de nivel medio, en este caso, Caltech. Pedí a dicho catedrático que expusiera las conclusiones a las que había llegado su universidad respecto a la pregunta de la diferencia entre espectros continuos y discontinuos, comenzando así el debate. En alguna ocasión pregunté a algún catedrático de otra universidad si estaba de acuerdo y si tenía algo que añadir. Otras veces, las preguntas de contraste se las iba haciendo a otros catedráticos, intentando que todas las universidades participaran. Me llevé una grata sorpresa cuando algunos catedráticos me solicitaban el turno de palabra para, por iniciativa propia, aportar algo a lo que ya habían dicho los compañeros. Al final, llegamos a los últimos 5 Ciclos de Mejora en el Aula (2020). Experiencias de Innovación Docente de la US 
minutos de clase debatiendo sobre el significado de los espectros discontinuos, por lo que, a modo de resumen y para que lo vieran de forma más gráfica, les puse una diapositiva con los saltos electrónicos, lo que creo que les ayudó mucho a visualizar el concepto del modelo atómico de Bohr y el motivo de la generación de los espectros discontinuos. Pero, de nuevo, me volví a quedar sin tiempo, de manera que el problema 4 se los tuve que enviar de nuevo por correo electrónico.

En general, la sensación ha sido muy buena, todos los alumnos que se realmente se han comprometido están muy integrados y participativos, tomándose las actividades muy en serio. Esto no quita que algunos de los que inicialmente quisieron participar en las actividades, se han echado un poco atrás. Yo creo que esto ha ocurrido al ver que solicito actividades diarias (aunque tienen 2 o 3 días entre clases para hacer dichas actividades) y no quieren tener la obligación de trabajar entre clase y clase. $\mathrm{O}$, al menos, no les compensa el trabajo con la "recompensa".

- Sesión del 16/11/2020: La sesión de hoy ha transcurrido, en líneas generales bastante bien. Los alumnos, de nuevo, han participado muy activamente, no sólo respondiendo a mis preguntas de contraste, sino para ayudar a otros compañeros, ya fuera para explicar mejor o más profundamente algún concepto, como cuando el compañero que estaba intentando exponer sus conclusiones se quedaba atorado en algo. Por ello, no sólo han intervenido los estudiantes de doctorado (seleccioné a estos alumnos para no seguir el mismo orden alfabético, para que no pudieran predecir quiénes van a intervenir en la sesión), sino que también han intervenido otros alumnos a los que en teoría no les "tocaba" participar. La única nota "negativa" que pondría sería la falta de tiempo, es decir, que el debate me ha requerido más tiempo del esperado, lo que me ha obligado a no realizar la actividad 4 de este problema (P.3). Yo creo Ciclos de Mejora en el Aula (2020). Experiencias de Innovación Docente de la US
Esta obra se distribuye con la licencia Creative Commons 
que realizar esta actividad hubiera sido necesaria para que les quedara más claro el significado de los números cuánticos. Aun así, puesto que la actividad 5 eran la resolución de más ejercicios, les he pedido que entreguen también los de la actividad 4. Además, puesto que la próxima sesión versará sobre la realización de ejercicios sobre números cuánticos, aprovecharé para volver a hacer las preguntas de contraste.

- Sesión del 19/11/2020: La sesión de hoy comprendía la realización de ejercicios, usándolos para seguir haciendo o repetir las preguntas de contraste. Aunque, en lineas generales, ha salido bien de nuevo (los alumnos han participado sin problema intentando resolver los ejercicios y justificando sus decisiones), el principal inconveniente ha sido el hecho de tener que hacerlo con una clase online. He tenido que usar la pizarra en blanco y, usando mi tableta gráfica, les he ido diciendo que me dijeran lo que yo tenía que escribir. Claro, esto ha provocado que la tendencia fuera que me dieran directamente el resultado final del ejercicio, así que yo les he tenido que ir pidiendo lo que hacían paso a paso. Esto ha significado que hemos tardado muchísimo en cada ejercicio y no nos ha dado tiempo a hacerlos todos. Además, ha significado que yo he tenido que estar interviniendo continuamente. Por lo tanto, debo plantearme la idoneidad de este tipo de actividad en tiempos de docencia online. La parte positiva de realizar este tipo de actividad basada en ejercicios, que ellos han tenido que realizar con anterioridad a la clase, es que, en comparación con otros años, los alumnos han pedido muchas más aclaraciones y han participado, incluso, en las aclaraciones que sus propios compañeros pedían. Espero que esto haya ocurrido por un mayor interés por su parte y se traduzca en un mejor aprendizaje...

- Sesión del 23/11/2020: La sesión de hoy ha transcurrido de forma muy similar a la anterior desde el punto de vista tecnológico: yo he conectado mi tableta y he

Ciclos de Mejora en el Aula (2020). Experiencias de Innovación Docente de la US Esta obra se distribuye con la licencia Creative Commons 
pedido a los alumnos de máster que actuaran de representantes, de manera que yo iba escribiendo lo que ellos me iban diciendo. La sesión estaba programada para tratar configuración electrónica a partir de los ejercicios del boletín. Además, puesto que en los días anteriores habían sido capaces de trabajar los conceptos sin que yo les anticipara nada, a última hora decidí no colgarles ningún vídeo, para ver hasta dónde podrían llegar por sí mismos. Sorpresivamente, la configuración electrónica la han captado muy bien y no han necesitado mucha ayuda para hacer los ejercicios. Pero no ha ocurrido lo mismo con los diagramas de energía, que ahí sí que he tenido que intervenir más y explicarlos en clase, porque andaban bastante perdidos. Quizá, en este último caso sí que hubiera sido conveniente pasarles algún vídeo o texto hablando de ellos antes de la clase, para que lo trabajaran.

Como aspecto a mejorar, que ha ocurrido en esta última clase y en la anterior, es que el hecho de no hacer ellos los ejercicios directamente, sino a través de mí, ha hecho que yo intervenga más de lo que debía, a veces porque me lo requerían, otras por instinto. Creo que se ha debido, en parte, a la forma de impartir la clase, en modo online, pero también ha influido mucho ese instinto que llevamos dentro de ser nosotros los que impartimos las clases, los que "explicamos" los ejercicios. Y, por supuesto, esto debo de evitarlo en futuras ocasiones.

\section{Evaluación del aprendizaje: Escaleras de Aprendizaje y Cuadro de Evolución}

Para evaluar la evolución del aprendizaje de los alumnos, estos realizan, al final de las sesiones, el mismo cuestionario que hicieron al principio del CIMA. En base a estos resultados, se prepara una escalera de aprendizaje para cada problema propuesto (Figura 3)

Ciclos de Mejora en el Aula (2020). Experiencias de Innovación Docente de la US Esta obra se distribuye con la licencia Creative Commons 


\section{BELÉN BEGINES RUIZ}

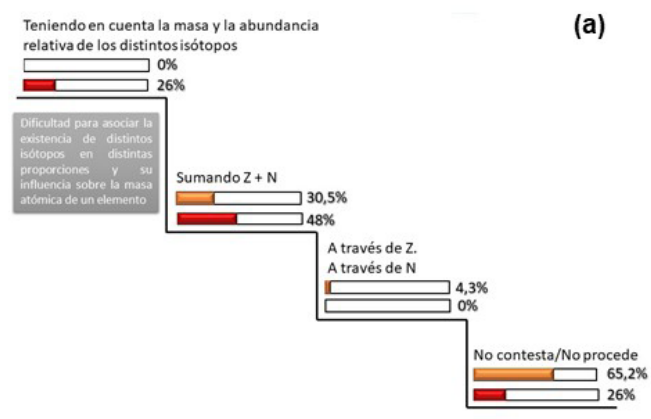

Alrededor del núcleo ubicándose en differentes órbitas, que determinan los distintosniveles de

(b)

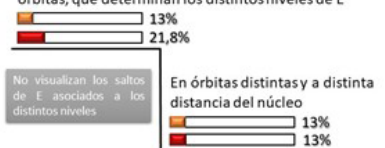

(b)

(c)

No, la E depende del nively el subnivelen el que se encuentren
los electrones. Los electrones que están en el mismo orbital si

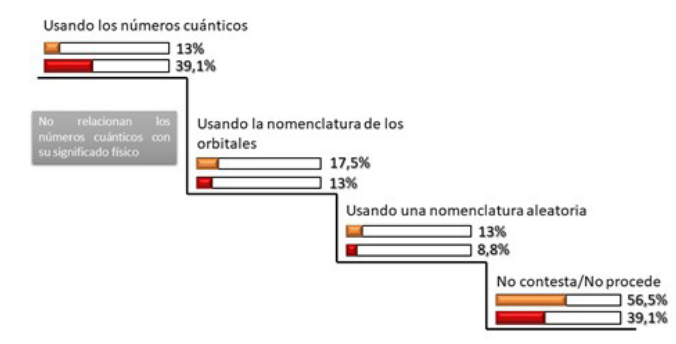
tienen iguale

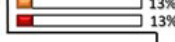
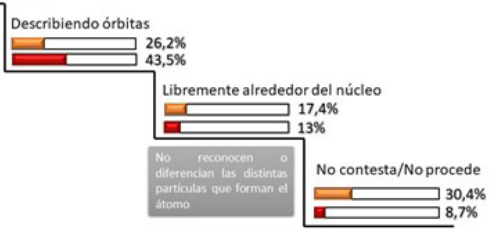

(d)

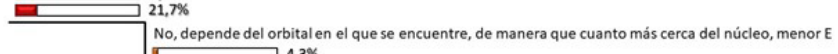
No, depende del orbita

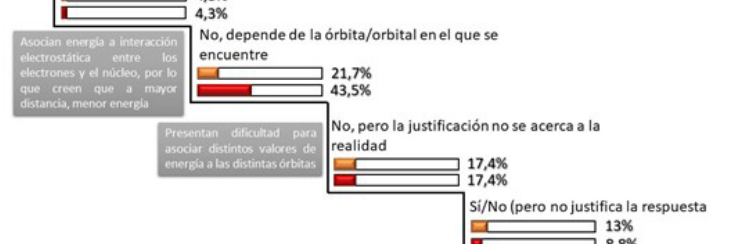

$\square$ Respuestas antes del CIMA

No contesta/No procede

Respuestas después del CIMA

Figura 3. Escaleras de aprendizaje obtenidas con el análisis de cuestionarios para los distintos problemas: (a) P.1, (b) P.2, (c) P.3 y (d) P.4

Ciclos de Mejora en el Aula (2020). Experiencias de Innovación Docente de la US cc (i) $\Theta$ Esta obra se distribuye con la licencia Creative Commons 
Como se puede observar, en la pregunta 1 se han obtenido resultados muy positivos, puesto que cerca del $40 \%$ de los estudiantes han dado un salto de dos escalones, desde el nivel a hasta el c. Además, un 26\% ha alcanzado el escalón más alto. Se puede apreciar también que el $48 \%$ de los alumnos se queda en el nivel c, ya que presentan una clara dificultad para asociar la existencia de distintos isótopos en distintas proporciones y su influencia sobre la masa atómica del elemento en cuestión.

Sin embargo, la pregunta 2 ha generado resultados más dispares. Aunque se puede apreciar que más del 20\% de los alumnos que se encontraban en el nivel a han dado un salto, si nos fijamos únicamente en los números, podemos ver que la mayor parte de los estudiantes se han quedado en el nivel c. En este caso, me temo que algunos de ellos hayan contestado al problema de manera escueta, sencilla, a pesar de que su conocimiento era mayor, ya que más de un alumno que estaba en niveles superiores han bajado hasta este nivel. Y si esto es así, los resultados son muy buenos, puesto que parte de estos estudiantes que están en el nivel c deberían, en realidad, subir al d o al e. En este caso, se reconocen dos obstáculos principales: entre el nivel a y el b, ya que los alumnos tienen dificultades para reconocer las distintas partículas que forman el átomo; y entre el d y el e, ya que no visualizan los saltos de energía asociados a los distintos niveles energéticos.

La pregunta 3 es la que ha arrojado unos resultados más polarizados. Por un lado, es en la que menor porcentaje de estudiantes han saltado del nivel a, solo un $17.4 \%$; sin embargo, es en la que un mayor porcentaje de alumnos, un 39.1\%, han alcanzado el nivel más alto, a pesar de que, para llegar a dicho nivel, muchos de ellos mostraron dificultades para relacionar los números cuánticos con su significado físico.

Respecto a la pregunta 4, los resultados también son muy satisfactorios, con sólo un $12.3 \%$ de alumnos que no han subido de los dos primeros escalones. Sin embargo, 
casi la mitad de los estudiantes se queda en el nivel d, ya que les cuesta mucho visualizar todo lo relacionado con la energía.

Así mismo, para hacer un estudio más pormenorizado de la evolución de cada alumno, se realizó un Cuadro de Evolución (Tabla 3) con 15 alumnos elegidos al azar:

Tabla 3. Cuadro de evolución de un conjunto de 15 alumnos elegidos al azar

\begin{tabular}{|c|c|c|c|c|c|c|c|c|c|c|c|c|c|c|}
\hline Alumno & P1-i & P1-f & $\Delta \mathrm{P} 1$ & P2-i & P2-f & $\triangle \mathrm{P} 2$ & P3-i & Pe-f & $\triangle \mathrm{P} 3$ & $P 4-i$ & P4-f & $\triangle \mathrm{P} 4$ & $\begin{array}{c}\text { Saltos } \\
\text { totales } \\
\text { por } \\
\text { alumnos }\end{array}$ & $\begin{array}{c}\text { Saltos } \\
\text { promedio }\end{array}$ \\
\hline 1 & C & $d$ & 1 & $d$ & C & -1 & C & $d$ & 1 & C & $f$ & 3 & 4 & 1 \\
\hline 2 & a & a & 0 & a & C & 2 & a & c & 2 & $b$ & $d$ & 2 & 6 & 1,5 \\
\hline 3 & C & c & 0 & a & e & 4 & a & a & 0 & $\mathrm{a}$ & $d$ & 3 & 7 & 1,75 \\
\hline 4 & $\mathrm{a}$ & $d$ & 3 & a & $b$ & 1 & a & C & 2 & C & C & 0 & 6 & 1,5 \\
\hline 5 & a & $d$ & 3 & C & e & 2 & a & $d$ & 3 & $b$ & $d$ & 2 & 10 & 2,5 \\
\hline 6 & $b$ & C & 1 & a & C & 2 & a & a & 0 & b & $f$ & 4 & 7 & 1,75 \\
\hline 7 & a & C & 2 & C & d & 1 & C & c & 0 & c & $d$ & 1 & 4 & 1 \\
\hline 8 & a & c & 2 & $\mathrm{~d}$ & $d$ & 0 & C & $d$ & 1 & $d$ & d & 0 & 3 & 0,75 \\
\hline 9 & C & $\mathrm{d}$ & 1 & C & C & 0 & a & $\mathrm{d}$ & 3 & a & $\mathrm{d}$ & 3 & 7 & 1,75 \\
\hline 10 & C & C & 0 & b & C & 1 & $b$ & $b$ & 0 & a & $d$ & 3 & 4 & 1 \\
\hline 11 & $\mathrm{a}$ & $d$ & 3 & $b$ & $d$ & 2 & $\mathrm{a}$ & a & 0 & C & c & 0 & 5 & 1,25 \\
\hline 12 & $a$ & C & 2 & a & e & 4 & a & a & 0 & a & C & 2 & 8 & 2 \\
\hline 13 & a & c & 2 & b & c & 1 & $b$ & b & 0 & a & $f$ & 5 & 8 & 2 \\
\hline 14 & a & c & 2 & $d$ & e & 3 & $d$ & d & 0 & C & $f$ & 3 & 8 & 2 \\
\hline 15 & C & $d$ & 1 & e & e & 0 & a & $d$ & 3 & e & $f$ & 1 & 5 & 1,25 \\
\hline $\begin{array}{c}\text { Saltos } \\
\text { totales }\end{array}$ & & & 23 & & & 22 & & & 15 & & & 32 & & \\
\hline
\end{tabular}

En la Tabla 3, se puede observar el estudio completo de la evolución del conjunto total de alumnos, ya que podemos apreciar el nivel inicial y final de cada estudiante

Ciclos de Mejora en el Aula (2020). Experiencias de Innovación Docente de la US Esta obra se distribuye con la licencia Creative Commons 
en cada problema, el número de saltos de nivel que el aprendizaje ha generado y el número de saltos totales en el conjunto. Así, se puede apreciar qué problema ha provocado una mayor modificación de los modelos mentales iniciales de los alumnos. Además, se puede también llevar a cabo un estudio pormenorizado de cada alumno, a través de la suma de saltos totales realizados en todos los problemas, así como el número de saltos promedio que ha tenido dicho estudiante.

\section{Evaluación del CIMA}

\section{Cuestiones a mantener y cambios a introducir para un futuro Ciclo de Mejora}

Personalmente, estoy muy satisfecha por cómo ha ido el CIMA, fundamentalmente por lo contento que están los alumnos. Destaco dos frases que me han escrito en un cuestionario anónimo. La primera sintetiza muy bien lo que han expresado la mayoría de los alumnos en este cuestionario: Me ha gustado mucho ya que me ha obligado a trabajar antes el contenido y ahora me veo con conocimientos suficientes para llevar bien el examen sin tener que estudiar y memorizar sin más, he llegado a entender de verdad lo dado. Me emociona pensar que, en muchos casos, he conseguido el objetivo de que los alumnos aprendan de verdad, que hayan adquirido los conocimientos principales interiorizándolos, modificando realmente su modelo inicial. La segunda: Me ha gustado mucho. Quisiera añadir, ya que esto es anónimo y no entrará como 'pelotería' que agradezco mucho su trabajo y dedicación para hacer nuestras clases más dinámicas y variadas. El mundo necesita más profesores entregados como usted. Creo que poco más hay que añadir cuando uno lee estas palabras, todo el trabajo y el esfuerzo compensa. Y, sobre todo, te da una idea de que estás en el camino correcto...

Ciclos de Mejora en el Aula (2020). Experiencias de Innovación Docente de la US Esta obra se distribuye con la licencia Creative Commons 
Por supuesto, hay aspectos que deben mejorar para un futuro CIMA. El primero, y más importante, es la planificación de las tareas. En todas las sesiones me he quedado corta, muy corta, de tiempo. En alguna de ellas he sido consciente de que los alumnos, aunque iban entendiendo los conceptos e interiorizándolos, necesitaban algo más de tiempo, alguna actividad de contraste más, para captar todos los conceptos en su conjunto para modificar, así su modelo inicial. Y esto me lleva al segundo aspecto a mejorar: mi participación en las sesiones. Por lo general, conforme iba acercándose la finalización de las sesiones mi participación iba en aumento, puesto que quería que se tocaran todos los conceptos y todas las preguntas de contraste planificadas, por lo que en las últimas no les dejaba el tiempo suficiente para que las pensaran detenidamente. Los alumnos iban, por decirlo de alguna manera, muy dirigidos por mí en las respuestas a estas últimas preguntas. Por lo tanto, debo planificar para que las actividades duren más tiempo y poder, así, darles más espacio a los alumnos. Por último, también debo mejorar la contextualización inicial para atraer más a los estudiantes. Debo tener en cuenta que la asignatura se imparte en el primer curso del Grado en Educación Primaria, fundamentalmente. Quizás este tipo de alumnos no se sientan muy atraídos por cuestiones que atañen únicamente al campo de la ciencia. Probablemente ellos ni siquiera hayan oído nunca nombrar la revista Nature y la implicación que, para nosotros los científicos, tendría publicar en ella. Aun así, han participado en las actividades unas 40 personas, que es casi un $60 \%$ de los estudiantes matriculados en mi grupo, lo cual creo que no está mal para ser la primera vez que intento llevar a cabo un CIMA. Pero tengo la sensación de que podría haber captado a muchos más si hubiera usado otro tipo de "gancho".

Ciclos de Mejora en el Aula (2020). Experiencias de Innovación Docente de la US Esta obra se distribuye con la licencia Creative Commons 


\section{Principios Didácticos argumentados}

La principal idea que me llevo de este curso es que el modelo metodológico a aplicar, si queremos obtener un aprendizaje efectivo de los alumnos, debe ser completamente diferente al modelo tradicional de clase magistral. El nuevo modelo debe estar basado en el trabajo del propio alumno, siendo el profesor el encargado de provocar la modificación del modelo mental inicial de los alumnos a través de sus preguntas/actividades de contraste. Así, el docente debe trabajar no para "explicar" bien los contenidos, sino para que los alumnos sean capaces de adoptar estos, interiorizándolos y modificando sus modelos mentales iniciales, puesto que es la única manera de que el aprendizaje sea realmente efectivo. Además, también he experimentado en primera persona como los propios alumnos reconocen, aunque sin saber exactamente por qué, que esta forma de aprendizaje es mucho mejor y más efectiva para ellos. Los estudiantes se fijan fundamentalmente en el hecho de que "tienen que estudiar menos" para el examen, pero esto es indicativo de que, efectivamente, el aprendizaje ha sido efectivo, que ha modificado su modelo mental inicial y que, por tanto, tienen que memorizar menos.

Una de las ideas que yo ya tenía clara en mi mente incluso antes de comenzar este curso, pero que se ha afianzado durante el mismo, es el considerar a los alumnos como personas cercanas, evitando situarnos por encima de ellos y mostrar autoridad. En mi opinión, esto no hace más que cohibirlos e impedir que se expresen libremente en clase, generando miedo o inseguridades. Por ello, me parece fundamental que el alumno sienta la libertad de expresarse con naturalidad durante una clase, lo que le llevará a una mejor interacción con sus compañeros y, sobre todo, con el profesor.

Además, he podido comprobar en primera persona cómo los alumnos aprenden mucho más cuando trabajan en grupo de iguales que cuando lo hacen de forma individual.

Ciclos de Mejora en el Aula (2020). Experiencias de Innovación Docente de la US Esta obra se distribuye con la licencia Creative Commons 
La aplicación de mi CIMA me ha ayudado a darme cuenta de que, con las horas de docencia que impartimos, es imposible tratar de forma efectiva absolutamente todos los contenidos que pretendemos con la enseñanza tradicional. Por ello, es esencial que modifiquemos los planes de estudio para ser capaces de reducirlos a los contenidos más importantes, los contenidos estructurantes, de manera que podamos tratarlos con suficiente profundidad como para que pasen a formar parte de los modelos mentales de los alumnos.

Por otra parte, también he experimentado la necesidad de preparar suficientemente bien y con la claridad necesaria tanto el contexto como las preguntas o problemas clave presentados a los alumnos para, no solo ser capaz de despertar su atención y curiosidad, sino para promover una serie de actividades que nos permitan generar los contrastes suficientes en los alumnos. Al igual que los profesores tendemos a acercarnos al modelo metodológico tradicional, puesto que es el que conocemos y en el que nos sentimos seguros, los alumnos también están acostumbrados a trabajar en este modelo, que les requiere menos esfuerzo y trabajo diario. Esto hace que, si el "gancho" propuesto no les atrae lo suficiente, tiendan a rendirse ante la solicitud del trabajo diario. Si no conseguimos esto, por un lado, los alumnos no se van a involucrar en las actividades y, por tanto, en la construcción de su propio aprendizaje; y por otro, como profesores, podemos tener muchas dificultades para enlazar las actividades de contraste correctas, necesarias y que nos permitan profundizar en todos los contenidos.

Igualmente, la secuencia de actividades programadas debe estar estudiada y planificada con detenimiento, al igual que las preguntas de contraste, para generar la necesidad en el alumno de replantearse su modelo mental inicial. Por ello, es importante que el profesor tenga muy claro cuáles son estas preguntas/actividades para no correr el riesgo de dejar parte del contenido sin tratar o a alumnos que no lo hayan entendido plenamente.

También resalto la importancia del diagnóstico inicial de los alumnos. Este nos permite determinar los 
principales obstáculos de aprendizaje, lo que, a su vez, nos ayuda a un diseño más óptimo, y acorde al nivel de conocimiento general de los alumnos, de las actividades/preguntas de contraste. Además, nos indica en qué parte del contenido tenemos que poner más énfasis y trabajar más concienzudamente.

Por último, me parece importante el diario de las sesiones, como la mejor manera de ir mejorando el diseño de las sesiones, de acuerdo con las experiencias vividas con anterioridad. Si esto no se hace, de un año a otro perderemos el recuerdo de las sensaciones, emociones o pequeños detalles experimentados durante dichas sesiones, perdiendo información importante para la mejora del CIMA.

Palabras clave: Fundamento de Ciencias de la Materia, Grado en Educación Primaria, Docencia Universitaria, Experimentación Docente Universitaria, Estructura del Átomo

Keywords: Matter Science, Primary Education Degree, University Teaching, University Teaching, Research, Atomic Structure

\section{Referencias bibliográficas}

Bain, K. (2008). Lo que hacen los mejores profesores universitarios. Valencia: Publicacions de la Universitat de Valencia .

Finkel, D. (2000). Dar Clase con la Boca Cerrada. Valencia: Publicacions de la Universitat de Valencia.

Porlán, R. (2017). Enseñanza universitaria: cómo mejorarla. Madrid: Ediciones Morata.

Ciclos de Mejora en el Aula (2020). Experiencias de Innovación Docente de la US Esta obra se distribuye con la licencia Creative Commons 